Research Paper

\title{
Papillary Urothelial Neoplasm of Low Malignant Potential (PUNLMP) After Initial TUR-BT: Comparative Analyses with Noninvasive Low-Grade Papillary Urothelial Carcinoma (LGPUC)
}

\author{
Jung Kwon Kim¹, Kyung Chul Moon², Chang Wook Jeong ${ }^{3}$, Cheol Kwak ${ }^{3}$, Hyun Hoe Kim³ , Ja Hyeon Ku ${ }^{3 凶}$ \\ 1. Department of Urology, Center for Prostate Cancer, Research Institute and Hospital of the National Cancer Center, Goyang, Korea; \\ 2. Department of Pathology, Seoul National University Hospital, Seoul, Korea; \\ 3. Department of Urology, Seoul National University Hospital, Seoul, Korea. \\ $\square$ Corresponding author: Ja Hyeon Ku, M.D. PhD., Professor, Department of Urology, Seoul National University Hospital, 101 Daehak-ro, Jongno-gu, Seoul \\ 110-744, Korea Tel: 82-2-2072-0361 Fax: 82-2-742-4665 E-mail: kuuro70@snu.ac.kr \\ (c) Ivyspring International Publisher. This is an open access article distributed under the terms of the Creative Commons Attribution (CC BY-NC) license \\ (https://creativecommons.org/licenses/by-nc/4.0/). See http://ivyspring.com/terms for full terms and conditions.
}

Received: 2017.03.09; Accepted: 2017.06.01; Published: 2017.08.24

\begin{abstract}
Purpose: To verify if the distinction between papillary urothelial neoplasm of low malignant potential (PUNLMP) and noninvasive low-grade papillary urothelial carcinoma (LGPUC) reflects a different biologic activity.

Materials and Methods: We reviewed and analyzed the clinical data from 678 patients who had a diagnosis of PUNLMP $(n=53)$ or noninvasive LGPUC $(n=625)$ after initial TUR-BT for bladder neoplasm between 2000 and 2012.

Results: The noninvasive LGPUC group showed a higher frequency of recurrence in comparison with the PUNLMP group $(46.7 \%$ vs. $30.2 \%, p=0.022)$. In contrast, there were no significant differences in progression $(15.2 \%$ vs. $18.9 \%, p=0.295)$ between the two groups. Grade progression was reported in 10 patients (LG: $n=5$; high grade: $n=2$; carcinoma in situ: $n=3$ ) and stage progression was reported in 2 patients (all: Tl) in PUNLMP group. The Kaplan-Meier survival analysis showed significantly decreased 5 -year recurrence-free survival (RFS) $(50.3 \%$ vs. $74.6 \%$, log-rank test, $p=0.014$ ) in the noninvasive LGPUC group compared to the PUNLMP group. However, there were no significant differences in progression-free survival (PFS) between the two groups. Multivariate analysis revealed that tumor grades according to $2004 \mathrm{WHO} / \mathrm{ISUP}$ classification system (PUNLMP vs. LG) were identified as significant predictors of RFS. However, it was not a significant predictor of both PFS and overall survival.

Conclusions: PUNLMP had a substantial number of recurrences (30.2\%), although RFS was better than noninvasive LGPUC. In addition, PUNLMP had a similar risk of progression compared with noninvasive LGPUC. Consequently, PUNLMP should be treated in a manner similar to noninvasive LGPUC, and long-term clinical follow-up should be recommended for patients with PUNLMP.
\end{abstract}

Key words: noninvasive low-grade papillary urothelial carcinoma; progression; PUNLMP; recurrence; urothelial carcinoma.

\section{Introduction}

Noninvasive papillary urothelial carcinoma (PUC) of the bladder is a major challenge in clinical setting due to its proclivity to recur or progress to invasive carcinoma [1-3]. Accordingly, the grading system of PUC has been a long-standing issue of debate. The 1973 World Health Organization (WHO) 
classification system originally included the categories from papilloma to grade 3 PUC [4]. However, arbitrary definitions including the intermediate grade (grade 2) were the major limitation of this system [5]. In 1998, the International Society of Urological Pathology (ISUP) proposed a new grading system [6]. This system introduced the term of papillary urothelial neoplasm of low malignant potential (PUNLMP), and this group of lesions included many cases that was categorized as grade 1 PUC in the 1973 WHO classification system [7]. Subsequently, the $2004 \mathrm{WHO} /$ ISUP classification system has been suggested for potential advantages over the 1973 WHO classification system, including more standardized histological criteria for grading the noninvasive PUC [8].

Although several previous studies confirmed the improvement of prognostic discrimination of this revised system [9-11], the other published comparisons had not clearly confirmed that the 2004 WHO/ISUP classification system has better reproducibility than the 1973 WHO classification system [3,12]. The most controversial issue of the 2004 WHO/ISUP classification system centers on the diagnosis of PUNLMP [7, 13-18]. Some of previous studies demonstrated that PUNLMP showed a low risk of recurrence and progression, and never resulted in cancer-specific death $[9,13,17]$. In contrast, several other studies indicated that patients with PUNLMP were at increased risk for recurrence and progression [14-16]. Subsequently, they recommended long-term clinical follow-up in these patients.

Thus the aim of this study was to verify if the distinction between PUNLMP and noninvasive low-grade papillary urothelial carcinoma (noninvasive LGPUC) reflects a different biologic activity.

\section{Materials and Methods}

\section{Ethics statement}

The Institutional Review Boards of the Seoul National University Hospital approved this study (Approval number: H-1612-004-809). As the present study was carried out retrospectively, written informed consent from patients was waived. Personal identifiers were completely removed and the data were analyzed anonymously. Our study was conducted according to the ethical standards laid down in the 1964 Declaration of Helsinki and its later amendments.

\section{Study cohort}

A total of 697 patients who had a diagnosis of PUNLMP or noninvasive LGPUC after initial TUR-BT for bladder neoplasm between 2000 and 2012 at our institution were included in this study. Clinical data in the medical records were retrospectively reviewed. Subsequently, patients with non-urothelial carcinoma history $(n=6)$, suspicious lymph node enlargement on radiologic evaluation $(\mathrm{n}=8)$, or incomplete information $(n=5)$ were excluded. Ultimately, 678 patients were included at the final analysis.

\section{Acquisition and definition of data}

Clinical preoperative variables included age, gender, body mass index (BMI), past medical history including gross hematuria (GHU) and upper urinary tract urothelial carcinoma (UTUC), tumor multiplicity ( 1 vs. $2-7$ vs. $\geq 8)$, and tumor size $(<3 \mathrm{~cm}$ vs. $\geq 3 \mathrm{~cm}$ ). Tumor size and number were obtained from cystoscopy measurement. The tumor stage and grade of all initial TUR-BT specimens were assessed by staff pathologists with genitourinary expertise according to the standard pathological procedure at our institution. Pathological staging was assigned to the 2010 TNM classification by the American Joint Committee on Cancer-Union for International Cancer Control (AJCC/UICC). Pathological grading was assessed according to both recommended the 1973 WHO classification system and/or the 2004 WHO/ISUP classification system. Repeat TUR-BT and intravesical chemotherapy (IVC) were performed at the discretion of the individual surgeons. Recurrence was defined as the histopathologically proven urothelial tumor relapse in the bladder or prostatic urethra during follow-up periods. Progression was defined as recurrence to a higher grade (LG, carcinoma in situ [CIS], high-grade [HG]) or stage $(\geq \mathrm{T} 1)$.

\section{Follow-up protocol}

According to the institutional standardized postoperative protocol, patients were generally followed up after TUR-BT at least every 3-4 months for the first 2 year, semiannually for the next 3 years, and then annually thereafter. Follow-up consisted of urinary cytology, cystoscopy, and biopsy of suspicious lesions. Computed tomography scan for the upper urinary tract evaluation was generally carried out at the initial diagnosis and thereafter conducted only in cases of tumor recurrence or suspicion. IVC, including maintenance instillations, were performed at the surgeon's discretion.

Cause of death was determined by the responsible physicians and death certificates. Overall survival (OS) was calculated from the date of TUR-BT to the date of last follow-up or death. Recurrence-free survival (RFS) was defined as the interval between the date of the TUR-BT and the time of the first tumor recurrence. Progression-free survival (PFS) was 
defined as the interval from the date of the TUR-BT to the date of disease progression.

\section{Statistical analyses}

The clinicopathological characteristics were compared between PUNLMP and noninvasive LGPUC using chi-squared test for categorical variables, and independent t-test or Mann-Whitney $U$ test for continuous variables. The Kaplan-Meier method was used to calculate survival estimates for OS, RFS and PFS. Also, log-rank test was used to conduct comparisons between the groups. Univariate and multivariate Cox-proportional hazard regression analyses were performed to evaluate significant variables associated with OS, RFS and PFS. The variables with $p$-value less than 0.2 obtained in the univariate analysis were included in the multivariate analysis, and the final model was derived using the backward selection method with an elimination criterion of p-value greater than 0.05 . In addition, we used the propensity score methodology [19] to control other confounding factors in analyzing survival outcomes. The propensity score matching analysis was conducted by using a nonparsimonious multiple logistic regression model. All statistical analyses were performed using commercially available software (IBM SPSS Statistics ver. 22.0, Armonk, NY, USA and the statistical package for R, ver. 2.13.2, R Foundation for Statistical Computing [http:/ / www.r-project.org]) and two-sided p-values $<0.05$ were considered statistically significant.

\section{Results}

Mean patient age was $70.2 \pm 12.2$ years, and the median follow-up period was 71.2 (range 0-205) months. At the last follow-up, there were 91 patients $(13.4 \%)$ who died of any cause, and 19 patients $(2.8 \%)$ died of bladder cancer. Among the patients, 308 $(45.4 \%)$ patients experienced recurrence. Stage and grade progression were reported in $78(11.5 \%)$ and 76 $(11.2 \%)$ patients, respectively. On pathologic review, $53(7.8 \%)$ and $625(92.2 \%)$ patients were diagnosed as PUNLMP and noninvasive LGPUC, respectively. IVC was given to 1 patient in the PUNLMP group (1.9\%; Mitomycin C [MMC]), and 32 patients in the noninvasive LGPUC group (5.1\%; MMC: $n=14$; Bacillus Calmette-Guérin [BCG]: $\mathrm{n}=18$ ).

\section{Comparison of clinicopathological features between two groups}

The comparative analysis of clinicopathological features between the PUNLMP group and the noninvasive LGPUC group are summarized in Table 1. The median follow-up periods were 73.9 (range, 3-165) and 70.5 (0-205) months in PUNLMP and noninvasive LGPUC group, respectively $(p=0.745)$. The noninvasive LGPUC group showed a higher frequency of GHU $(73.6 \%$ vs. $45.3 \%, \mathrm{p}<0.001)$ and recurrence $(46.7 \%$ vs. $30.2 \%, \mathrm{p}=0.022)$ in comparison with the PUNLMP group.

Table 1. Comparative analysis results of variables between PUNLMP group and noninvasive LGPUC group after initial TUR-BT.

\begin{tabular}{llll}
\hline & PUNLMP (n=53) & LGPUC $(\mathrm{n}=625)$ & p-value \\
\hline Age, $\geq 60$ & $38(71.7 \%)$ & $516(82.6 \%)$ & 0.063 \\
Gender, male & $41(77.4 \%)$ & $515(82.4 \%)$ & 0.354 \\
BMI, mean (SD) & $24.06(2.48)$ & $24.07(3.02)$ & 0.981 \\
GHU, yes & $24(45.3 \%)$ & $460(73.6 \%)$ & $<0.001$ \\
1973 WHO classification, & & & $<0.001$ \\
grade & & & \\
1 & $53(100 \%)$ & $154(30.0 \%)$ & \\
2 & $0(0 \%)$ & $359(70.0 \%)$ & \\
Number & & & 0.509 \\
1 & $40(75.5 \%)$ & $430(68.8 \%)$ & \\
$2-7$ & $12(22.6 \%)$ & $188(30.1 \%)$ & \\
$\geq 8$ & $1(1.9 \%)$ & $7(1.1 \%)$ & \\
Size, cm & & & 0.810 \\
$\geq 3$ & $4(7.5 \%)$ & $64(10.2 \%)$ & \\
Repeat TUR-BT, yes & $0(0 \%)$ & $12(1.9 \%)$ & 0.613 \\
Intravesical therapy, yes & $1(1.9 \%)$ & $32(5.1 \%)$ & 0.742 \\
Upper urinary tract & $0(0 \%)$ & $8(1.3 \%)$ & 1.000 \\
recurrence, yes & & & \\
Recurrence, yes & $16(30.2 \%)$ & $292(46.7 \%)$ & 0.022 \\
Progression, yes & $10(18.9 \%)$ & $95(15.2 \%)$ & 0.295 \\
All cause death, yes & $6(11.3 \%)$ & $85(13.6 \%)$ & 0.834 \\
Cancer specific death, yes & $0(0 \%)$ & $19(3 \%)$ & 0.434 \\
\hline
\end{tabular}

BMI: body mass index, GHU: gross hematuria, PUNLMP: papillary urothelial neoplasm of low malignant potential, noninvasive LGPUC: noninvasive low-grade papillary urothelial carcinoma, WHO: World Health Organization

Median time to first recurrence were 9 (range, 3-103) and 11 (2-147) months in PUNLMP and noninvasive LGPUC group, respectively. In contrast, there were no significant differences in progression $(15.2 \%$ vs. $18.9 \%, p=0.295)$ between the two groups. Grade progression was patients (all: T1) in PUNLMP group. Two patients who had stage progression also reported grade progression (LG: $n=1 ; H G: n=1$ ). In noninvasive LGPUC group, there were 6 patients progressed to T2 and $19(3 \%)$ patients died of the disease. None of the patients in the PUNLMP group progressed to $\mathrm{T} 2$ or died of the disease.

\section{Survival outcomes and Cox-proportional hazard regression analyses: Propensity score matching analysis}

In the total study cohort, the Kaplan-Meier survival analysis showed significantly decreased 5 -year RFS (50.3\% vs. $74.6 \%$, log-rank test, $\mathrm{p}=0.014)$ in the noninvasive LGPUC group compared to the PUNLMP group (Fig. 1A.). However, there were no significant differences in PFS and OS between two groups (Fig. 1B. and 1C.). 
The propensity scores were computed by logistic regression modeling, with the independent variables which showed significant differences between two groups in the comparative analysis (Table 1): GHU and the 1973 WHO grade classification. A 1:1 matching ratio was used in our propensity analysis. Consequently, two groups were well balanced, and there were no significant differences between two groups in any of variables used for propensity score matching (Table S1). Subsequently, the Kaplan-Meier survival analysis was conducted, and consistently showed significantly decreased 5-year RFS $(31.0 \%$ vs. $74.6 \%$, log-rank test, $\mathrm{p}<0.001)$ in the noninvasive LGPUC group compared to the PUNLMP group (Fig. S2A). Notably, there were still no significant differences in PFS and OS between two groups (Fig. S2B and S2C.).

A

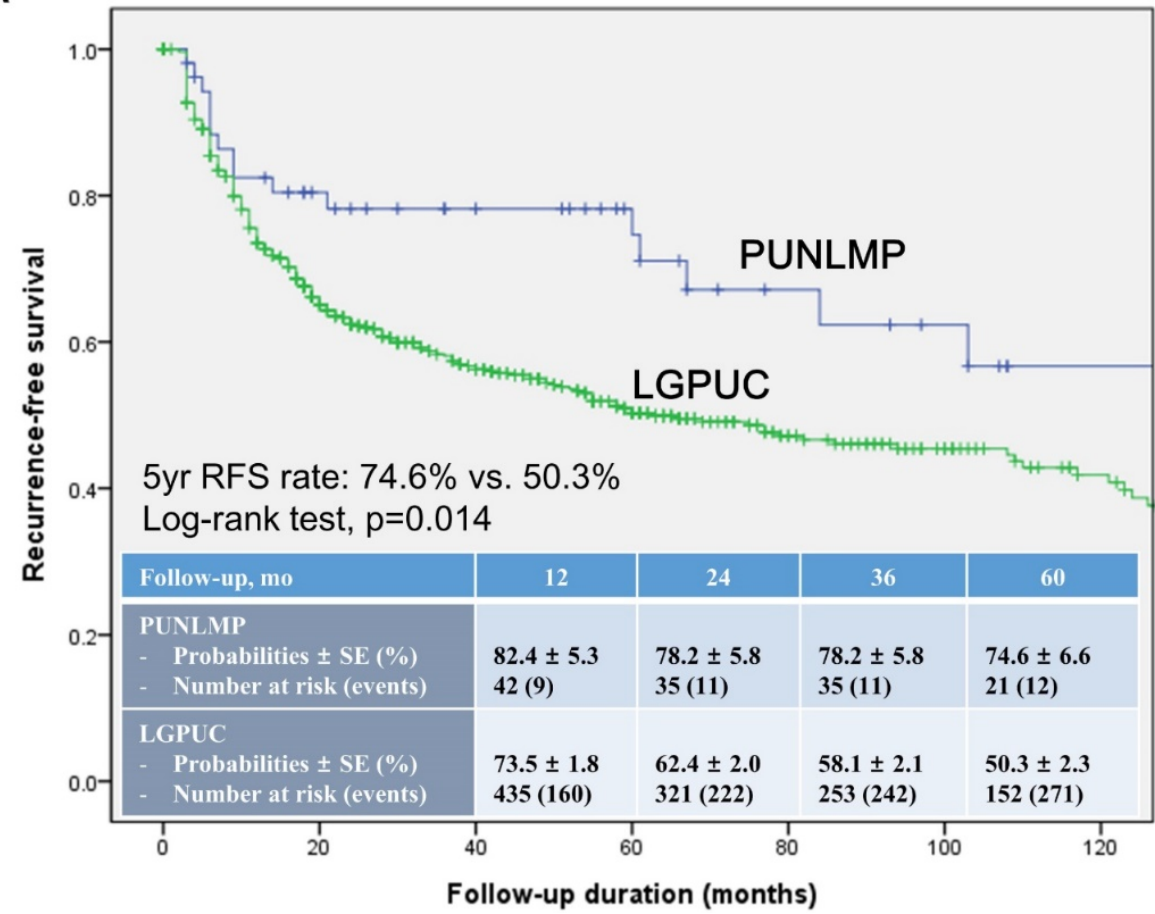

B

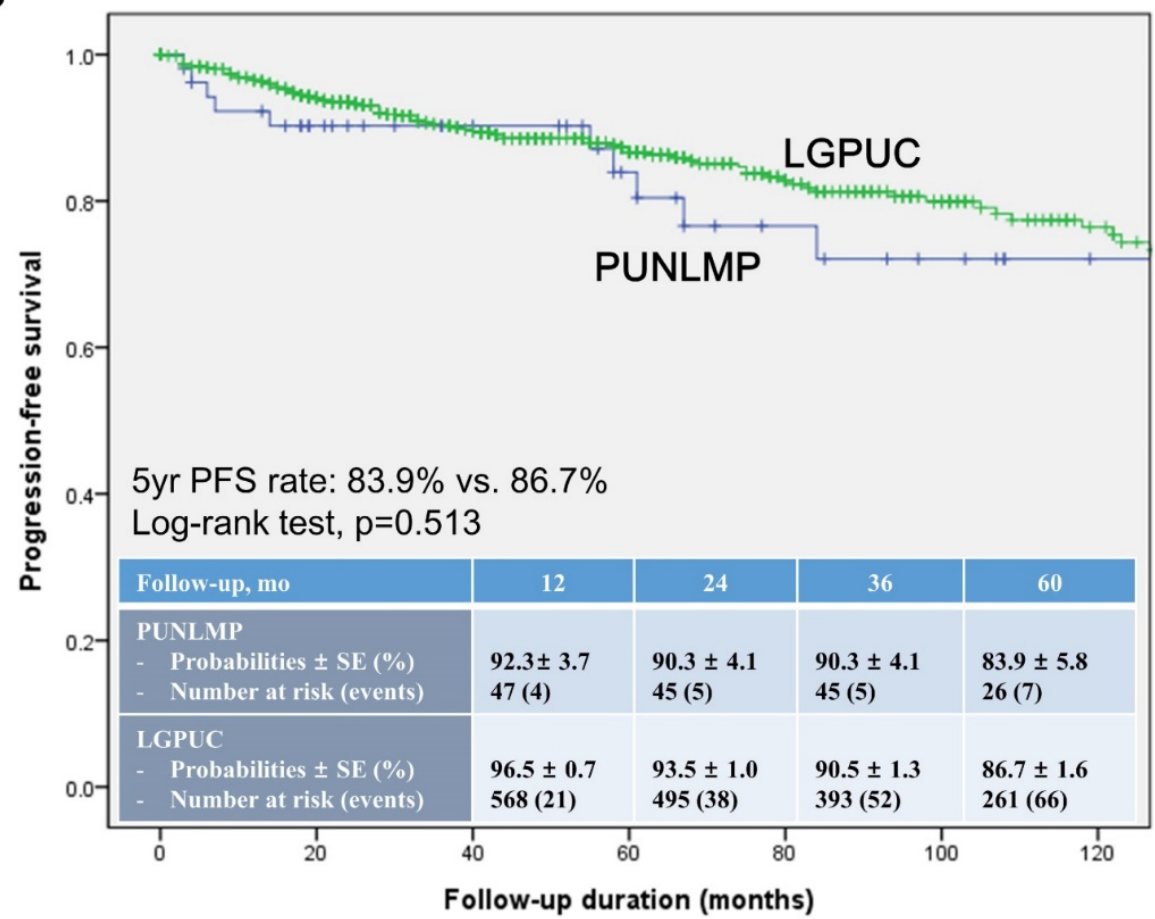




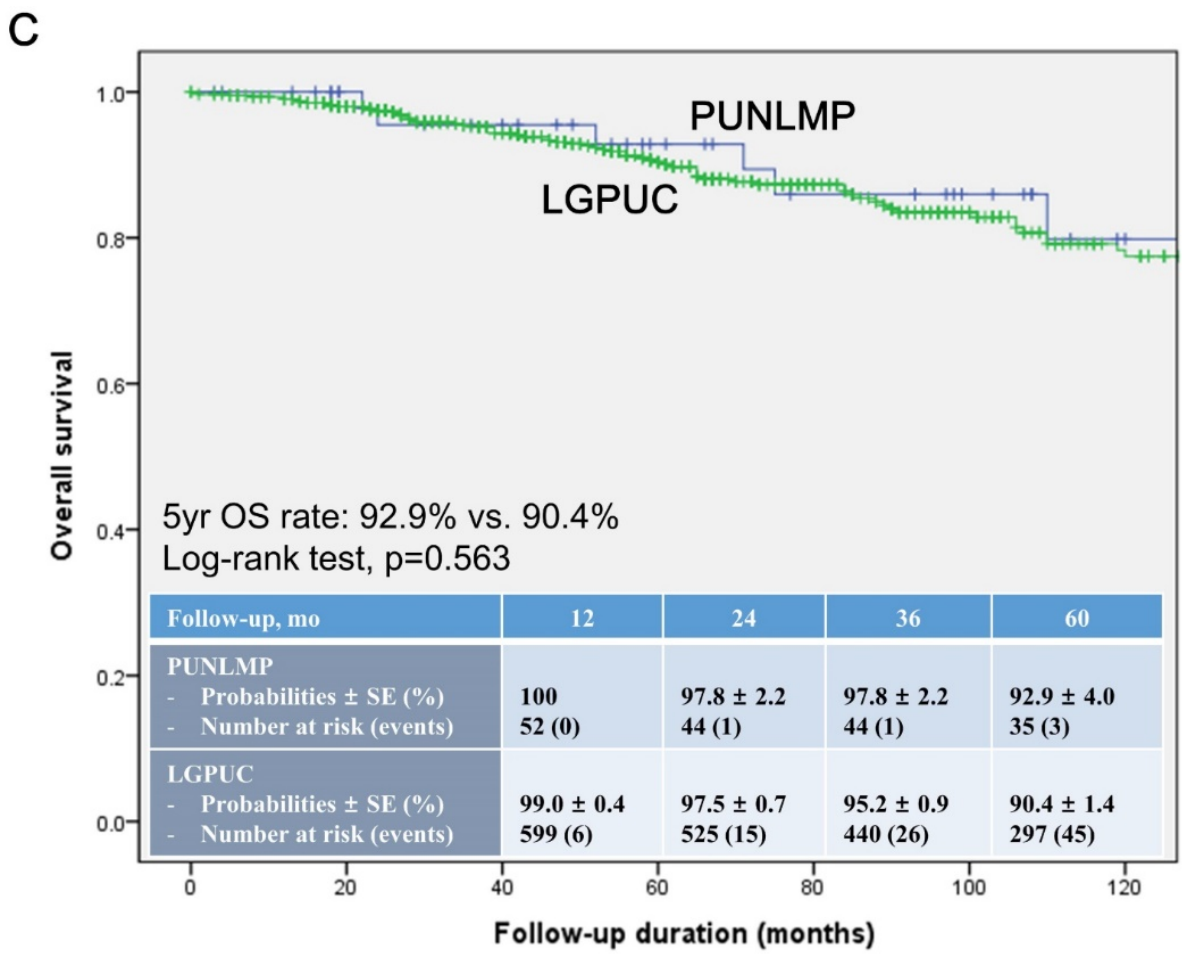

Figure 1. Kaplan-Meier survival curves for (A) recurrence-free survival (RFS), (B) progression-free survival (PFS), and overall survival (C) for patients with papillary urothelial neoplasm of low malignant potential (PUNLMP) and noninvasive low-grade papillary urothelial carcinoma (noninvasive LGPUC) according to the 2004 WHO/ISUP classification system.

Table 2 summarized the results of multivariate Cox regression analyses based on the total study cohort. Multivariate analysis revealed that GHU $(p=0.007)$, tumor grades according to the 2004 WHO/ISUP classification system $(p=0.042)$ and tumor number $(\mathrm{p}<0.001)$ were identified as significant predictors of RFS. In addition, age $(p=0.016)$ and $B M I$ $(\mathrm{P}<0.001)$ were identified as significant predictors of OS, and tumor number $(\mathrm{p}=0.024)$ was identified as significant predictor of PFS. Whereas, tumor grades according to the 2004 WHO/ISUP classification system was not a significant predictor of both PFS and OS.

\section{Discussion}

Both in the 1973 WHO classification system and $2004 \mathrm{WHO} /$ ISUP classification system, PUCs were divided into four categories. At first, the definition of papilloma was same in the both 1973 WHO classification system and 2004 WHO/ISUP classification system. At the other end of the grading category, all 1973 WHO grade 3 tumors were assigned to the HG carcinoma category in the 2004 WHO/ISUP classification system. Whereas, there was no direct matched translation to the 2004 WHO/ISUP classification system for 1973 WHO grades 1 and 2 carcinomas. The grade 1 carcinomas according to the 1973 WHO classification system, in case of showing no or minimal cytological atypia and merely thickened urothelium with, at most, nuclear enlargement, are reclassified as PUNLMP $[8,16]$. The other 1973 WHO grade 1 tumors with definite yet slight cytological atypia are reassigned to the LG category in the $2004 \mathrm{WHO} /$ ISUP classification system $[8,16]$. Subsequently, considering the interobserver variability and the prognostic accuracy among LG category tumors, it is obvious that morphology alone would be insufficient to predict the clinical outcome of the disease $[3,14]$.

PUNLMP was created as category of neither benign nor intrinsically malignant neoplasm in the 2004 WHO/ISUP classification system [8]. Accordingly, PUNLMP should not be regarded as cancer, which has psychosocial and financial significance. However, several previous studies showed that PUNLMPs could recur in a substantial proportion of patients [9, 14-18, 20-22]. Notably, some studies also represented that PUNLMPs had a low, but definitive risk of progression to a higher grade or stage lesions [14-16, 18]. Subsequently, the criticisms evolved that PUNLMP should be qualified as a carcinoma and that all PUC should be categorized as two groups (LG and HG) $[4,8]$. In addition, several studies demonstrated that a low level of agreement in the histologic distinction, or no significant prognostic differences between PUNLMP and noninvasive LGPUC [14-16, 22].

Current study showed that noninvasive LGPUC 
group showed a higher frequency of recurrence in comparison with the PUNLMP group $(46.7 \%$ vs. $30.2 \%, p=0.022$, Table 1). The Kaplan-Meier survival analysis also showed significantly decreased 5-year RFS (50.3\% vs. $74.6 \%$, log-rank test, $p=0.014)$ in noninvasive LGPUC group compared to the PUNLMP group (Fig. 1A.). In concordance with previous studies [9, 13-17, 20-22], substantial number of patients reported recurrence even with a distinct risk between two groups. In addition, median time to first recurrence were even shorter in PUNLMP group than noninvasive LGPUC group (9 vs. 11 months).

In contrast, current study showed no significant differences in progression $(18.9 \%$ vs. $15.2 \%, p=0.295)$ between two groups. The Kaplan-Meier survival analysis also showed no significant differences in PFS between two groups (Fig. 1B.). In concordance with some previous studies [14-16], in terms of progression, PUNLMP and noninvasive LGPUC were similar with a definitive risk proportion. The high progression rates in current study may be derived from the definition that we used. Some previous studies defined progression as a recurrence with HG and/or invasive PUC [8]. Our definition, whereas, was broader and also included recurrent to LG or CIS. The rationale for our definition was derived from previous studies, which demonstrated that the prognosis of LGPUC was worse than that of PUNLMP [3, 13, 14, 23]. Notably, there were 2 patients who reported stage progression to pT1 in current study. Consequently, our findings further support the notion that patients with PUNLMP have increased risks of recurrence, progression, and even cancer-specific death [14-16].

To the best of our knowledge, the current study is by far the largest single-institutional study comparing the PUNLMP and noninvasive LGPUC. In addition, our institutional pathologist had reported both the 1973 WHO classification system and 2004 WHO/ISUP classification system. Accordingly, current study showed that all PUNLMPs were also reported 1973 WHO grade 1 (Table 1). Whereas, 30\% of LGPUC were reported as 1973 WHO grade 1, and the other $70 \%$ were reported as $1973 \mathrm{WHO}$ grade 2 (Table 1). The Kaplan-Meier survival analysis showed significantly decreased 5 -year RFS ( $46.1 \%$ vs. $58.7 \%$, log-rank test, $\mathrm{p}=0.016$ ) in grade 2 group compared to grade 1 group (Fig. S1A). In contrast, there were no significant differences in PFS between two groups (Fig. S1B). Consequently, the majority of patients with LGPUC showed distinct histology from PUNLMP according to the $1973 \mathrm{WHO}$ classification system; both LGPUC and PUNLMP showed no significant differences in terms of PFS.

There are several well-known parameters including tumor number, tumor size, and molecular markers on the clinical outcomes for PUC [3, 24, 25]. Current study showed that tumor number ( 1 vs. $\geq 2$ ) was the significant predictor for both RFS and PFS in multivariate Cox regression analyses; however, tumor size was not observed as a significant predictor (Table $2)$. It might be derived from a small number of patients $(n=68,10.0 \%)$ who reported tumor size of $\geq$ $3 \mathrm{~cm}$.

Table 2. Multivariate Cox regression analyses results for evaluating variables associated with overall survival (OS), recurrence-free survival (RFS), and progression-free survival (PFS).

\begin{tabular}{|c|c|c|c|c|c|c|}
\hline & \multicolumn{2}{|l|}{ OS } & \multicolumn{2}{|l|}{ RFS } & \multicolumn{2}{|l|}{ PFS } \\
\hline & HR (95\% CI) & p-value & HR $(95 \%$ CI $)$ & p-value & HR $(95 \%$ CI) & p-value \\
\hline \multicolumn{7}{|l|}{ Age (years) } \\
\hline$<60$ & Reference & & Reference & & Reference & \\
\hline$\geq 60$ & $4.136(1.302-13.137)$ & 0.016 & $1.268(0.920-1.749)$ & 0.147 & $0.985(0.538-1.806)$ & 0.962 \\
\hline BMI & $0.851(0.789-0.918)$ & $<0.001$ & $0.990(0.953-1.028)$ & 0.587 & $0.999(0.933-1.070)$ & 0.982 \\
\hline GHU & $0.834(0.515-1.349)$ & 0.459 & $1.461(1.108-1.928)$ & 0.007 & $0.879(0.571-1.354)$ & 0.559 \\
\hline \multicolumn{7}{|c|}{$2004 \mathrm{WHO} /$ ISUP classification, grade } \\
\hline PUNLMP & Reference & & Reference & & Reference & \\
\hline Low grade & $1.277(0.556-2.936)$ & 0.565 & $1.537(1.032-2.556)$ & 0.042 & $0.843(0.439-1.619)$ & 0.609 \\
\hline \multicolumn{7}{|c|}{ Tumor number } \\
\hline 1 & Reference & & Reference & & Reference & \\
\hline$\geq 2$ & $1.211(0.775-1.892)$ & 0.401 & $1.956(1.554-2.462)$ & $<0.001$ & $1.611(1.066-2.436)$ & 0.024 \\
\hline \multicolumn{7}{|c|}{ Tumor size, $\mathrm{cm}$} \\
\hline$<3$ & Reference & & Reference & & Reference & \\
\hline$\geq 3$ & $1.338(0.726-2.464)$ & 0.350 & 1.037 (0.714-1.507) & 0.847 & $1.248(0.726-2.684)$ & 0.380 \\
\hline
\end{tabular}


The current study has several limitations. Due to the retrospective nonrandomized design, the conduction of repeat TUR-BT or IVC was at the discretion of the individual clinicians, not the standardized criteria. In addition, we did not address the issue of interobserver discrepancy, but rather depended on our pathologist with an expertise to identify and report according to standardized procedures of our institution for the purpose of interpretative consistency. Consequently, the current study was based on the single-institutional data and reflects real clinical practice. Finally, we did not include some molecular markers which have been evaluated for the differential diagnosis of LGPUC [21, 23-25]. However, previous studies have not clearly confirmed their usefulness or clinical implications yet [14].

In conclusion, current study showed that PUNLMP had lower risks of recurrence than noninvasive LGPUC, but with a substantial number of patients $(30.2 \%)$. In addition, in terms of progression, PUNLMP and noninvasive LGPUC were similar with a definitive risk proportion. Consequently, PUNLMP should be treated in a manner similar to noninvasive LGPUC, and long-term clinical follow-up would be recommended in these patients.

\section{Supplementary Material}

Supplementary figures and tables. http://www.jcancer.org/v08p2885s1.pdf

\section{Acknowledgments}

This work was supported by the National Research Foundation of Korea (NRF) grant funded by the Korea government (MSIP) (No. 2016R1A2B4011623).

\section{Competing Interests}

The authors have declared that no competing interest exists.

\section{References}

1. Montie JE, Abrahams NA, Bahnson RR, et al. Bladder cancer. Clinical guidelines in oncology. J Natl Compr Canc Netw. 2006; 4: 984-1014.

2. Hall MC, Chang SS, Dalbagni G, et al. Guideline for the management of nonmuscle invasive bladder cancer (stages Ta, T1, and Tis): 2007 update. J Urol. 2007; 178: 2314-30.

3. May M, Brookman-Amissah S, Roigas J, et al Prognostic Accuracy of Individual Uropathologists in Noninvasive Urinary Bladder Carcinoma: A Multicentre Study Comparing the 1973 and 2004 World Health Organisation Classifications. Eur Urol. 2010; 57: 850-8.

4. MacLennan GT, Kirkali Z, Cheng L. Histologic grading of noninvasive papillary urothelial neoplasms. Eur Urol. 2007; 51: 889-97.

5. Babjuk $M$, Burger $M$, Zigeuner $R$, et al. EAU Guidelines on Non-Muscle-invasive Urothelial Carcinoma of the Bladder: Update 2013. Eur Urol. 2013; 64: 639-53.

6. Epstein JI, Amin MB, Reuter VR, et al. The World Health Organization/International Society of Urological Pathology consensus classification of urothelial (transitional cell) neoplasms of the urinary bladder. Am J Surg Pathol. 1998; 22: 1435-48.
7. Campbell PA, Conrad RJ, Campbell CM, et al. Papillary urothelial neoplasm of low malignant potential: reliability of diagnosis and outcome. BJU Int. 2004; 93: 1228-31.

8. Miyamoto $\mathrm{H}$, Miller JS, Fajardo DA, et al. Non-invasive papillary urothelial neoplasms: the 2004 WHO/ISUP classification system. Pathol Int. 2010; 60: 1-8.

9. Holmäng $\mathrm{S}$, Hedelin $\mathrm{H}$, Anderstrom $\mathrm{C}$, et al. Recurrence and progression in low grade papillary urothelial tumors. J Urol. 1999; 162: 702-7.

10. Cheng L, Neumann RM, Nehra A, et al. Cancer heterogeneity and its biologic implications in the grading of urothelial carcinoma. Cancer. 2000; 88: 1663-70.

11. Cheng L, Neuman RM, Weaver AL, et al. Grading and staging of bladder carcinoma in transurethral resection specimens. Correlation with 105 matched cystectomy specimens. Am J Clin Pathol. 2000; 113: 275-9.

12. van Rhijn BW, van Leenders GJ, Ooms BC, et al. The pathologist's mean grade is constant and individualizes the prognostic value of bladder cancer grading. Eur Urol. 2010; 57: 1052-7.

13. Pan CC, Chang YH, Chen KK, et al. Prognostic Significance of the 2004 WHO/ISUP Classification for Prediction of Recurrence, Progression, and Cancer-Specific Mortality of Non-Muscle-Invasive Urothelial Tumors of the Urinary Bladder: a clinicopathologic study of 1,515 cases. Am J Clin Pathol. 2010; 133: 788-95.

14. Lee TK, Chaux A, Karram S, et al. Papillary urothelial neoplasm of low malignant potential of the urinary bladder: clinicopathologic and outcome analysis from a single academic center. Hum Pathol. 2011; 42: 1799-803.

15. Cheng L, Neumann RM, Bostwick DG. Papillary urothelial neoplasms of low malignant potential. Clinical and biologic implications. Cancer. 1999; 86: 2102-8

16. Jones TD, Cheng L. Papillary Urothelial Neoplasm of Low Malignant Potential: Evolving Terminology and Concepts. J Urol. 2006; 175: 1995-2003.

17. Pich A, Chiusa L, Formiconi A, et al. Biologic differences between noninvasive papillary urothelial neoplasms of low malignant potential and low-grade (grade 1) papillary carcinomas of the bladder. Am J Surg Pathol. 2001; 25: 1528-33.

18. Samartunga H, Makarov DV, Epstein JI. Comparison of WHO/ISUP and WHO classification of noninvasive papillary urothelial neoplasms for risk of progression. Urology. 2002; 60: 315-9.

19. D'Agostino RB Jr. Propensity score methods for bias reduction in the comparison of a treatment to a non-randomized control group. Stat Med 1998; 17: 2265-81.

20. Herr HW, Donat SM, Reuter VE. Management of low grade papillary bladder tumors. J Urol. 2007; 178: 1201-5.

21. Pich A, Chiusa L, Formiconi A, et al. Proliferative activity is the most significant predictor of recurrence in noninvasive papillary urothelial neoplasms of low malignant potential and grade 1 papillary carcinomas of the bladder. Cancer. 2002; 95: 784-90.

22. Ramos D, Navarro S, Villamón R, et al. Cytokeratin expression patterns in low-grade papillary urothelial neoplasms of the urinary bladder. Cancer. 2003; 97: 1876-83.

23. Holmäng $\mathrm{S}$, Andius $\mathrm{P}$, Hedelin $\mathrm{H}$, et al. Stage progression in Ta papillary urothelial tumors: relationship to grade, immunohistochemical expression of tumor markers, mitotic frequency and DNA ploidy. J Urol. 2001; 165: 1124-8.

24. Sylvester RJ, van der Meijden AP, Oosterlinck W, et al. Predicting recurrence and progression in individual patients with stage Ta T1 bladder cancer using EORTC risk tables: a combined analysis of 2596 patients from seven EORTC trials. Eur Urol. 2006; 49: 466-77.

25. Burger $M$, van der Aa MN, van Oers JM, et al. Prediction of progression of non-muscle-invasive bladder cancer by WHO 1973 and 2004 grading and by FGFR3 mutation status: a prospective study. Eur Urol. 2008; 54: 835-43. 